DOI 10.4171/JEMS/248

Angelo Alvino · Vincenzo Ferone · Carlo Nitsch

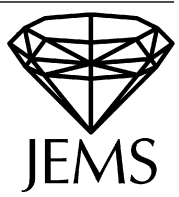

\title{
A sharp isoperimetric inequality in the plane
}

Received June 15, 2008 and in revised form March 24, 2009

\begin{abstract}
We show that among all the convex bounded domains in $\mathbb{R}^{2}$ having a fixed Fraenkel asymmetry index, there exists only one convex set (up to similarity) which minimizes the isoperimetric deficit. We also show how to construct this set. The result can be read as a sharp improvement of the isoperimetric inequality for convex planar domains.
\end{abstract}

Keywords. Isoperimetric inequality, Bonnesen-style inequality, Fraenkel asymmetry, isoperimetric deficit

\section{Introduction}

The classical isoperimetric inequality in the plane states that, among all the subsets of $\mathbb{R}^{2}$ of prescribed finite measure, the disk has the smallest perimeter, that is,

$$
P(E) \geq(4 \pi|E|)^{1 / 2}, \quad \text { with equality if and only if } E \text { is a disk. }
$$

Here $|E|$ and $P(E)$ denote, as usual, the measure and the perimeter of the set $E \subset \mathbb{R}^{2}$.

It is almost impossible to give exhaustive references concerning the isoperimetric inequality, therefore we refer the reader to some pioneering papers [3, 6, 19, 22], to the paper by De Giorgi [10] in the general framework of finite perimeter sets in $\mathbb{R}^{n}$, to the reviews [14, 23, 28] and to the books [8, 9].

In [4, 5] Bonnesen introduced some remarkable inequalities which imply the isoperimetric one (see also the reviews [8, 24]). For example, we recall that for bounded convex planar sets he proved that

$$
P(E)^{2}-4 \pi|E| \geq 4 \pi d^{2} .
$$

Here $d$ is the thickness of the minimal annulus containing the boundary of $E$. The chief tool in the proof was a symmetrization technique known as annular symmetrization. Later Bonnesen's work led to the study of a wider class of inequalities nowadays known as Bonnesen-style isoperimetric inequalities (see [8, 24]).

A. Alvino, V. Ferone, C. Nitsch: Dipartimento di Matematica e Applicazioni "R. Caccioppoli", Università degli Studi di Napoli "Federico II", Complesso Monte S. Angelo, via Cintia, 80126 Napoli, Italy; e-mail: angelo.alvino@unina.it; ferone@unina.it; c.nitsch@unina.it

Mathematics Subject Classification (2010): 52A40, 52A10, 49Q10 
If we define the isoperimetric deficit of a bounded set $E$ by

$$
\Delta P(E)=\frac{P(E)}{(4 \pi|E|)^{1 / 2}}-1,
$$

the isoperimetric inequality becomes

$$
\Delta P(E) \geq 0, \quad \text { with equality if and only if } E \text { is a disk. }
$$

Following Osserman [8, 24] a Bonnesen-style isoperimetric inequality in general can be written in the form

$$
\Delta P(E) \geq F(E),
$$

where the function $F$ is nonnegative, vanishes only on the disks, and somehow measures how much $E$ deviates from a disk. There are many different kinds of functions $F$ satisfying these properties, and each one leads to a different refinement of the standard isoperimetric inequality. In this paper we are interested in those functions $F$ whose dependence on the set $E$ is only through the so-called Fraenkel asymmetry index, i.e.

$$
\alpha(E)=\min _{x \in \mathbb{R}^{2}} \frac{\left|E \backslash D_{R}(x)\right|}{|E|},
$$

where $D_{R}(x)$ is the disk centered at $x$ and having the same measure as $E$. Both the Fraenkel asymmetry index and the isoperimetric deficit have the property of being invariant under similarities.

A remarkable Bonnesen-style inequality valid for convex planar sets was provided by Hall and Hayman [17]. They proved an inequality which in terms of isoperimetric deficit and Fraenkel asymmetry index reads

$$
\Delta P(E) \geq \frac{\pi}{2(4-\pi)} \alpha(E)^{2}-c_{0} \alpha(E)^{3},
$$

where the constant $\pi /(2(4-\pi))$ is optimal while $c_{0}$ is an unknown nonnegative constant.

For completeness we mention that, in the last two decades, Bonnesen-style inequalities were found in higher dimensions and for very general sets, as well as in the anisotropic case, for example in [11, 12, 13, 15, 16]. In particular, Hall [16] proved that the isoperimetric deficit of any smooth open set in $\mathbb{R}^{n}$ is bounded from below by a constant times a certain power of the Fraenkel asymmetry index. The fact that the optimal power is 2 (regardless of the dimension) has been recently proved in [15].

As far as the authors know, in the literature the investigation of $(1.1)$ is confined to functions $F$ expressed as powers of $\alpha(E)$. Still several problems are left unsolved. In particular, according to [15],

$$
\inf _{E} \frac{\Delta P(E)}{\alpha(E)^{2}}
$$

is positive in the class of measurable sets in $\mathbb{R}^{n}$, but still unknown. Indeed, even for convex planar domains, it was unclear whether this constant coincided or not with $\pi /(2(4-\pi))$ in 1.3. As a consequence of our result, we give an answer to this question in Remark 2.1 
In the present paper we provide a sharp Bonnesen-style inequality for planar convex domains involving just $\Delta P(E)$ and $\alpha(E)$. Obviously there exists a maximal function $G$ such that

$$
\Delta P(E) \geq G(\alpha(E)) .
$$

The determination of the function $G$ is somehow connected with the investigation of the shape of the optimal sets, i.e., those sets which achieve equality in (1.4). We show that, for any $0 \leq \alpha_{0}<1$, it is possible to compute $G\left(\alpha_{0}\right)$. In particular, we work out an analytic expression for the set $E$ with asymmetry index $\alpha(E)=\alpha_{0}$ which achieves equality in (1.4). Moreover we prove that such a set is unique up to similarity. Our result is based on a new symmetrization technique closely related to circular symmetrization [20], and well suited to the bidimensional framework. Using this tool we show how to reshape a given planar convex set keeping, step by step, its measure and its Fraenkel asymmetry index fixed and shortening the perimeter. The procedure eventually provides the family of optimal sets. We explicitly remark that, in order to keep the Fraenkel asymmetry index fixed, the convexity assumption on $E$ is needed and that it is not clear how to get rid of it.

\section{Main statement}

In order to formulate our main statement we begin by defining the family $\mathfrak{S}$ of optimal sets. This family contains the circles and any convex set $S$ satisfying the following properties:

- $S$ is symmetric with respect to two orthogonal axes (following [15] we shall refer to this property as 2-symmetry);

- $S$ has a smooth $C^{1}$ boundary made of four circular arcs $\left\{a_{i}\right\}_{1 \leq i \leq 4}$, two of which can possibly degenerate into parallel segments;

- $\alpha(S)=|S \backslash D| /|D|, D$ being the disk having the same measure as $S$ and centered at the intersection of the axes of symmetry of $S$;

- whenever $a_{i}$ is a proper circular arc (for some $1 \leq i \leq 4$ ) then it does not $\operatorname{cross} \partial D$, namely either $a_{i} \subset \bar{D}$ or $a_{i} \subset \mathbb{R}^{2} \backslash D$.

For the sake of completeness we give an explicit analytic expression for the family $\mathfrak{S}$. If the symmetry axes are used as reference axes in the $(\xi, \eta)$-plane, then up to similarity, such a family can be described as a one-parameter family $\left\{H_{\vartheta}\right\}_{\vartheta \in(0, \pi / 4]}$ where $\left|H_{\vartheta}\right|=\pi$. We set $H_{\pi / 4} \equiv D_{1}(0)$ while for $\vartheta \neq \pi / 4$ the parameter $\vartheta$ denotes the angular coordinate of the intersection of $\partial H_{\vartheta}$ with $\partial D$ in the first quadrant of the $(\xi, \eta)$-plane. When $0<$ $\vartheta \leq \arctan (\pi / 4)$ the part of the set $H_{\vartheta}$ which lies in the first quadrant is described as (see Figure 1)

$$
\begin{aligned}
\left\{(\xi, \eta) \in H_{\vartheta}: \xi \geq\right. & 0, \eta \geq 0\} \\
& =\left\{(\xi, \eta) \in \mathbb{R}^{2}: 0 \leq \xi \leq \frac{\pi}{4} \frac{\cos ^{2} \vartheta}{\sin \vartheta}+\sin \vartheta, 0 \leq \eta \leq \gamma_{1}(\xi)\right\},
\end{aligned}
$$




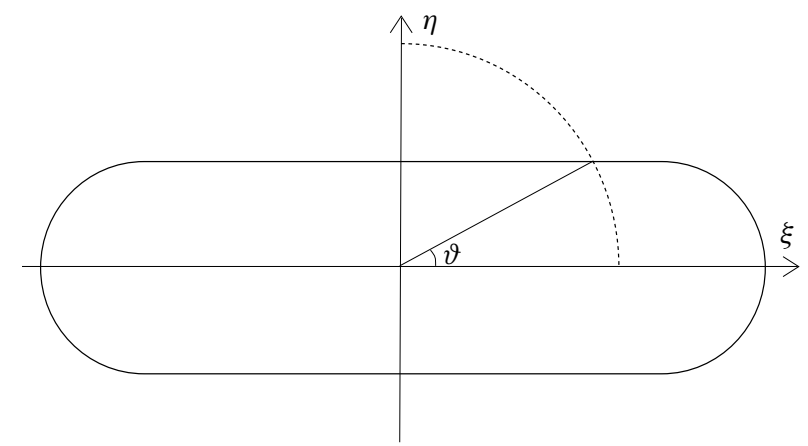

Fig. 1. The set $H_{\vartheta}$ for $0<\vartheta \leq \arctan (\pi / 4)$. The dashed line indicates the portion of $\partial D$ lying in the first quadrant of the $(\xi, \eta)$-plane.

where

$$
\gamma_{1}(\xi)= \begin{cases}\sin \vartheta & \text { if } 0 \leq \xi \leq \frac{\pi}{4} \frac{\cos ^{2} \vartheta}{\sin \vartheta} \\ \sqrt{\sin ^{2} \vartheta-\left(\xi-\frac{\pi}{4} \frac{\cos ^{2} \vartheta}{\sin \vartheta}\right)^{2}} & \text { if } \frac{\pi}{4} \frac{\cos ^{2} \vartheta}{\sin \vartheta}<\xi \leq \frac{\pi}{4} \frac{\cos ^{2} \vartheta}{\sin \vartheta}+\sin \vartheta .\end{cases}
$$

In this case we have

$$
\Delta P\left(H_{\vartheta}\right)=\frac{(1-\sin \vartheta)^{2}}{2 \sin \vartheta}, \quad \alpha\left(H_{\vartheta}\right)=\frac{\pi-2 \vartheta-2 \sin \vartheta \cos \vartheta}{\pi} .
$$

We observe that, when $\vartheta \rightarrow 0, H_{\vartheta}$ degenerates into a segment and

$$
\lim _{\vartheta \rightarrow 0} \Delta P\left(H_{\vartheta}\right)=+\infty, \quad \lim _{\vartheta \rightarrow 0} \alpha\left(H_{\vartheta}\right)=1 .
$$

When $\arctan (\pi / 4)<\vartheta<\pi / 4$ the part of the set $H_{\vartheta}$ which lies in the first quadrant is bounded by the arcs of circumferences which meet at the point $(\cos \vartheta, \sin \vartheta)$ in such a way that $\partial H_{\vartheta}$ is of class $C^{1}$. This means that the radii of such circumferences which pass through the point $(\cos \vartheta, \sin \vartheta)$ belong to the same straight line which forms an angle $x$ with the $\eta$-axis (see Figure 2).

For this type of domain the isoperimetric deficit is given by

$$
\Delta P\left(H_{\vartheta}\right)=\frac{2}{\pi}\left(\left(\frac{\sin \vartheta}{\cos x}\left(\frac{\pi}{2}-x\right)+\frac{\cos \vartheta}{\sin x} x\right)-\frac{\pi}{2}\right),
$$

while the Fraenkel asymmetry is given by

$$
\alpha\left(H_{\vartheta}\right)=\frac{2}{\pi}\left(\frac{\sin ^{2} \vartheta}{\cos ^{2} x}\left(\frac{\pi}{2}-x-\sin x \cos x\right)-\vartheta+\sin \vartheta \cos \vartheta\right) .
$$




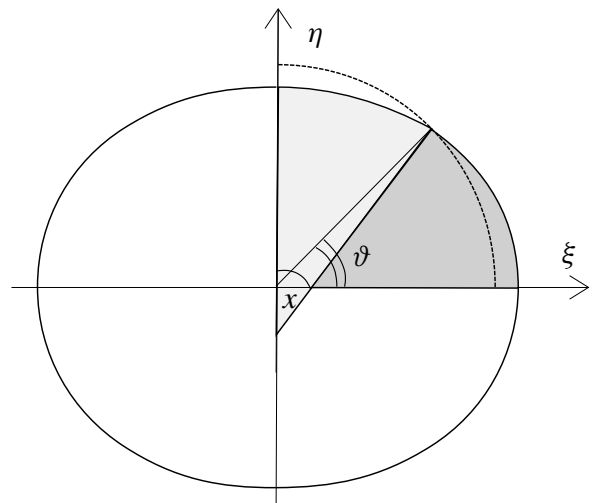

Fig. 2. The set $H_{\vartheta}$ for $\arctan (\pi / 4)<\vartheta<(\pi / 4)$. The dashed line indicates the portion of $\partial D$ laying in the first quadrant of the $(\xi, \eta)$-plane.

The condition $\left|\Omega_{\vartheta}\right|=\pi$ gives a relation between $x$ and $\vartheta$ :

$$
\frac{\sin ^{2} \vartheta}{\cos ^{2} x}\left(\frac{\pi}{2}-x\right)+\frac{\cos ^{2} \vartheta}{\sin ^{2} x} x-\frac{(\cos \vartheta-\tan x \sin \vartheta)^{2}}{\tan x}=\frac{\pi}{2} .
$$

As a result of a straightforward but very tedious calculation both $\Delta P\left(H_{\vartheta}\right)$ and $\alpha\left(H_{\vartheta}\right)$ turn out to be continuous decreasing functions of $\vartheta$ and therefore the following property holds.

Proposition 2.1. For each $0 \leq t<1$, up to similarity, there exists a unique set $S_{t} \in \mathfrak{S}$ such that $\alpha\left(S_{t}\right)=t$. Moreover, whenever $0 \leq t_{1}<t_{2}<1$, then $\Delta P\left(S_{t_{1}}\right)<\Delta P\left(S_{t_{2}}\right)$.

Therefore we can parametrize the family $\mathfrak{S}$ by the Fraenkel asymmetry index or the isoperimetric deficit. The statement of our main result follows.

Theorem 2.1. Every convex set $\Omega \subset \mathbb{R}^{2}$ satisfies

$$
\Delta P(\Omega) \geq \Delta P\left(S_{\alpha(\Omega)}\right),
$$

where equality holds if and only if $\Omega \in \mathfrak{S}$.

Remark 2.1. By using the monotonicity of $\Delta P\left(H_{\vartheta}\right)$ and $\alpha\left(H_{\vartheta}\right)$ with respect to $\vartheta$ we deduce the monotonicity of the function $G$ in (1.4) but regretfully it seems impossible to write its expression in an elementary form. Nevertheless inequality (1.3) can be obtained by an asymptotic expansion of $\alpha\left(H_{\vartheta}\right)$ and $\Delta P\left(H_{\vartheta}\right)$ in the limit as $\vartheta \rightarrow \pi / 4$. In particular it is quite easy to deduce from 2.1 -2.3 that

$$
\lim _{\vartheta \rightarrow \pi / 4} \frac{\Delta P\left(H_{\vartheta}\right)}{\alpha\left(H_{\vartheta}\right)^{2}}=\frac{\pi}{2(4-\pi)} .
$$

What is more interesting is that, for each $0<\bar{\vartheta}<\pi / 4$, we have

$$
\min _{\bar{\vartheta} \leq \vartheta \leq \pi / 4} \frac{\Delta P\left(H_{\vartheta}\right)}{\alpha\left(H_{\vartheta}\right)^{2}}<\frac{\pi}{2(4-\pi)} \simeq 1.83 \text {. }
$$


The above statements can be proved, for example, using the fact that from 2.3 the variable $\vartheta$ can be explicitly written in terms of $x$, namely,

$$
\tan \vartheta=\frac{1}{\tan x} \frac{\pi \sin ^{2} x-2 x+2 \sin x \cos x}{2 x-\pi \sin ^{2} x+2 \sin x \cos x} .
$$

It follows, after a long but straightforward calculation, that the following expansion holds as $\vartheta \rightarrow \pi / 4$ :

$$
\frac{\Delta P\left(H_{\vartheta}\right)}{\alpha\left(H_{\vartheta}\right)^{2}}=\frac{\pi}{2(4-\pi)}-\frac{\pi^{3}(16-5 \pi)(14-3 \pi)}{24(4-\pi)^{4}(\pi-2)} \alpha\left(H_{\vartheta}\right)^{2}+O\left(\alpha\left(H_{\vartheta}\right)^{4}\right) .
$$

Here we have given an expansion to the second order, but any order can be obtained. The observation that the coefficient of the second order term is negative immediately gives (2.5) and (2.6). Furthermore, we can say that 1.3 does not hold true for $c_{0}=0$ (regardless of how "small" $\Delta P(E)$ and $\alpha(E)$ are).

By a numerical calculation we can also obtain the following estimate:

$$
\min _{0<\vartheta \leq \pi / 4} \frac{\Delta P\left(H_{\vartheta}\right)}{\alpha\left(H_{\vartheta}\right)^{2}} \simeq 1.62 .
$$

Finally we propose a different interpretation of Theorem 2.1

Corollary 2.1. For any given $0 \leq \Delta P_{0}<+\infty$ let $0 \leq \bar{t}<1$ be such that

$$
\Delta P\left(S_{\bar{t}}\right)=\Delta P_{0} .
$$

Then for all convex sets $\Omega \subset \mathbb{R}^{2}$ with $\Delta P(\Omega)=\Delta P_{0}$ we have

$$
\alpha(\Omega) \leq \alpha\left(S_{\bar{t}}\right) .
$$

Therefore, taking into account the meaning of the asymmetry index, our result provides a sharp control on how much a convex set differs from the disk having the same measure in terms of its isoperimetric deficit.

\section{Rearrangements and pseudo-circular symmetrization}

We first recall the definition of symmetric decreasing and symmetric increasing rearrangements of functions, which will be used throughout the paper (see also [1, 2, 18, 27]). Let $f$ be a positive measurable function defined in $(a, b)$. If $\mu_{f}(t)=|\{s \in(a, b): f>t\}|$ is the distribution function of $f$, the decreasing rearrangement of $f$ is

$$
f^{*}(s)=\sup \left\{t \geq 0: \mu_{f}(t)>s\right\}, \quad s \in(0, b-a) .
$$

The symmetric decreasing and symmetric increasing rearrangements of $f$ are

$$
\begin{array}{rlrl}
f^{\sharp}(s) & =f^{*}(2|s|), & & s \in\left(-\frac{b-a}{2}, \frac{b-a}{2}\right), \\
f_{\sharp}(s) & =f^{*}(b-a-2|s|), & s \in\left(-\frac{b-a}{2}, \frac{b-a}{2}\right) .
\end{array}
$$


Given a coordinate system $(\xi, \eta)$ in $\mathbb{R}^{2}$, as usual the polar coordinates will be denoted by $(r, \theta)$ with $r \geq 0$ and $\theta \in[0,2 \pi]$, such that $\theta=0$ indicates the positive direction of the $\xi$-axis. According to Kawohl [20], a compact domain $\Omega \subset \mathbb{R}^{2}$ in polar coordinates can be Steiner symmetrized with respect to the angular coordinate $\theta$ to obtain a set $\Omega^{*}$ that is symmetric in $\theta$ and that he calls the circular symmetrized of $\Omega$. If we deal with a smooth and starshaped set with respect to the origin $O$, then the circular symmetrization can also be obtained by the Schwarz symmetrization of its radial function $\rho(\theta)$, which is the function that represents the boundary of the set in polar coordinates. From well-known properties of symmetrization we get $\left|\Omega^{*}\right|=|\Omega|$ and $\left|\partial \Omega^{*}\right| \leq|\partial \Omega|$.

Our goal is to introduce a new kind of symmetrization which somehow generalizes the circular one and which we shall call pseudo-circular symmetrization. Let us consider a smooth and bounded set $\Omega \subset \mathbb{R}^{2}$, starshaped with respect to the origin $O$, and two directions $\nu_{1}$ and $\nu_{2}$. We fix a coordinate system such that in polar coordinates $\theta=0$ is the direction of $\nu_{1}$ and denote by $\bar{\theta} \in[0,2 \pi]$ the direction of $\nu_{2}$. Next we consider the restriction $\rho_{1}(\theta)$ of $\rho(\theta)$ to the set $[0, \bar{\theta}]$ and the restriction $\rho_{2}(\theta)$ of $\rho(\theta)$ to the set $[\bar{\theta}, 2 \pi]$, and we define

$$
\begin{aligned}
& \bar{\rho}(\theta)= \begin{cases}\rho_{1}^{\sharp}(\theta-\bar{\theta} / 2), & \theta \in[0, \bar{\theta}), \\
\rho_{2}^{\sharp}(\theta-\pi-\bar{\theta} / 2), & \theta \in[\bar{\theta}, 2 \pi),\end{cases} \\
& \underline{\rho}(\theta)= \begin{cases}\rho_{1 \sharp}(\theta-\bar{\theta} / 2), & \theta \in[0, \bar{\theta}), \\
\rho_{2 \sharp}(\theta-\pi-\bar{\theta} / 2), & \theta \in[\bar{\theta}, 2 \pi) .\end{cases}
\end{aligned}
$$

Roughly speaking, we are considering the rearrangement of $\rho$ obtained by the symmetric rearrangements of its restrictions $\rho_{1}$ and $\rho_{2}$. A similar rearrangement can be found in [21].

Let $\Omega^{v_{1} \nu_{2}}$ and $\Omega_{v_{1} v_{2}}$ be the two open sets defined by the interior points of $\{(r, \theta)$ : $0 \leq r \leq \bar{\rho}(\theta)\}$ and $\{(r, \theta): 0 \leq r \leq \rho(\theta)\}$. They are the two possible pseudo-circular symmetrizations of $\Omega$. Obviously $\left|\Omega^{v_{1} \bar{v}_{2}}\right|=\left|\Omega_{v_{1} v_{2}}\right|=|\Omega|$ but in general $\bar{\rho}$ and $\rho$ are discontinuous at both 0 and $\bar{\theta}$, a condition which may increase the perimeter of the symmetrized set. The rest of this section is devoted to establishing sufficient conditions for either $\left|\partial \Omega^{v_{1} v_{2}}\right| \leq|\partial \Omega|$ or $\left|\partial \Omega_{v_{1} v_{2}}\right| \leq|\partial \Omega|$.

We first introduce the following definition.

Definition 3.1 (The set $\Lambda(a, b)$ ). We say that a function $g$ belongs to $\Lambda(a, b)$ if

- $g$ is a non-negative Lipschitz continuous function on $[a, b]$;

- \#\{g=t\} $\geq 2$ whenever $\min _{x \in[a, b]} g(x)<t<\max _{x \in[a, b]} g(x)$.

The main result of this section follows.

Lemma 3.1. Let $\Omega$ be an open starshaped set with respect to $O$, having a Lipschitz continuous radial function $\rho(\theta)$. Let $v_{1}, v_{2}$ and $\bar{\theta}$ be defined as before. If the restrictions of $\rho(\theta)$ to $(0, \bar{\theta})$ and $(\bar{\theta}, 2 \pi)$ belong to $\Lambda(0, \bar{\theta})$ and $\Lambda(\bar{\theta}, 2 \pi)$ respectively, and

$$
\min _{\theta \in(0, \bar{\theta})} \rho=\min _{\theta \in(\bar{\theta}, 2 \pi)} \rho \quad\left(\text { resp. } \max _{\theta \in(0, \bar{\theta})} \rho(\theta)=\max _{\theta \in(\bar{\theta}, 2 \pi)} \rho(\theta)\right),
$$


then $\bar{\rho}(\theta)$ (resp. $\underline{\rho}(\theta))$ is Lipschitz continuous, and $\left|\partial \Omega^{\nu_{1} \nu_{2}}\right| \leq|\partial \Omega|\left(\right.$ resp. $\left|\partial \Omega_{\nu_{1} \nu_{2}}\right| \leq$ $|\partial \Omega|)$.

Lemma 3.1 is a consequence of a well known result involving symmetric rearrangements [7, 26].

Lemma 3.2. If a function $g$ belongs to $\Lambda(a, b)$ then

$$
\begin{aligned}
& \int_{a}^{b} g(s)^{2} d s=\int_{-(a+b) / 2}^{(a+b) / 2} g^{\sharp}(s)^{2} d s=\int_{-(a+b) / 2}^{(a+b) / 2} g_{\sharp}(s)^{2} d s, \\
& \int_{a}^{b} \sqrt{g(s)^{2}+g^{\prime}(s)^{2}} d s \geq \int_{-(a+b) / 2}^{(a+b) / 2} \sqrt{g^{\sharp}(s)^{2}+g^{\sharp}(s)^{2}} d s, \\
& \int_{a}^{b} \sqrt{g(s)^{2}+g^{\prime}(s)^{2}} d s \geq \int_{-(a+b) / 2}^{(a+b) / 2} \sqrt{g_{\sharp}(s)^{2}+g_{\sharp}^{\prime}(s)^{2}} d s .
\end{aligned}
$$

Equality holds in (3.1) if and only if it holds in (3.2). In that case, if in addition we assume that $\left|\left\{s \in(a, b): g^{\sharp \prime}(s)=0\right\}\right|=0$ then

- $g(a)=g(b)=\min _{[a, b]} g$ if and only if $g=g^{\sharp}$;

- $g(a)=g(b)=\max _{[a, b]} g$ if and only if $g=g_{\sharp}$.

\section{Preliminary results}

Let $\Omega$ be an open bounded and convex subset of $\mathbb{R}^{2}$, and $D$ be a circle of radius $R=$ $(|\Omega| / \pi)^{1 / 2}$ that achieves the index of asymmetry of $\Omega$, i.e.

$$
|\Omega \backslash D|=\min _{x \in \mathbb{R}^{2}}\left|\Omega \backslash D_{R}(x)\right| .
$$

We refer to the last condition as the optimality condition for $D$ with respect to $\Omega$. From now on we shall use the center $O$ of $D$ as the origin of the coordinate system in $\mathbb{R}^{2}$. Since $\Omega$ is starshaped with respect to $O$ (it is easy to check that $O \in \Omega$, and a convex set is starshaped with respect to any interior point) there exists a one-to-one correspondence $\Phi: \partial D \rightarrow \partial \Omega$ such that, for any $x \in \partial D$, we have $x /|x|=\Phi(x) /|\Phi(x)|$. Throughout the paper we shall use the following notation (see Figure 3):

- $A=\partial D \cap \Omega$;

- $B=\partial D \backslash \bar{\Omega}$;

- $G=\partial D \cap \partial \Omega=\partial D \backslash(A \cup B)$;

- $\alpha_{i}(i \in \mathfrak{I} \equiv\{1,2, \ldots\} \subseteq \mathbb{N})$ are the connected components of $A$;

- $\beta_{k}(k \in \mathfrak{K} \equiv\{1,2, \ldots\} \subseteq \mathbb{N})$ are the connected components of $B$;

- $L$ denotes the arclength of a curve in $\mathbb{R}^{2}$;

- $T_{\nu}$ is the line containing $O$ and orthogonal to the direction $v$. As $T_{v}$ splits $\mathbb{R}^{2}$ in two we denote by $P_{v}^{+}$and $P_{v}^{-}$the resulting two open half-planes ( $v$ belongs to $P_{v}^{+}$);

- $\Pi_{\nu}$ is the projection operator on $T_{\nu}$. 


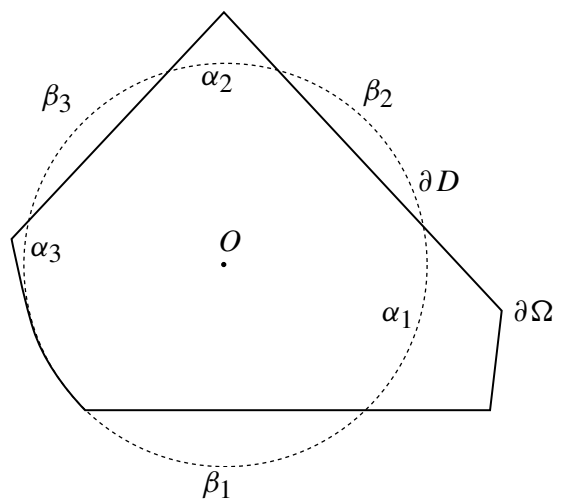

Fig. 3. A convex set $\Omega$ and a disk $D$ such that $|\Omega|=|D|$ and $\alpha(\Omega)=|\Omega \backslash D| /|D|$.

Let us now define $h_{\alpha_{i}}=\max \left\{|\Phi(x)|: x \in \alpha_{i}\right\}$ and $d_{\beta_{k}}=\min \left\{|\Phi(x)|: x \in \beta_{k}\right\}$. We shall assume without loss of generality that for every $i \in \mathfrak{I}$ and $k \in \mathfrak{K}$,

$$
\begin{aligned}
h_{\alpha_{i}} & =\max _{j \geq i} h_{\alpha_{j}}, \\
d_{\beta_{k}} & =\min _{j \geq k} d_{\beta_{j}},
\end{aligned}
$$

that is, just as in Figure 3, we assume that $\alpha_{i}$ and $\beta_{k}$ are ordered according to a "descending height order" in the radial direction. The existence of the maximum and the minimum in (4.2.1) and 4.2.ii) is a consequence of the fact that $\Omega$ is convex. Indeed, for any $C>0$ there exist only a finite number of indices $j$ such that $h_{\alpha_{j}} \geq R+C$ and a finite number of $j$ such that $d_{\beta_{j}} \leq R-C$.

In the following five lemmas we derive necessary conditions for the optimality of $D$ in 4.1 .

Lemma 4.1. Let $v$ be any direction in $\mathbb{R}^{2}$. Then

$$
L\left(\Pi_{v}\left(A \cap P_{v}^{+}\right)\right) \leq L\left(\Pi_{v}\left(A \cap P_{v}^{-}\right)\right)+L\left(\Pi_{v}\left(G \cap P_{v}^{-}\right)\right) .
$$

Proof. We denote by $\hat{n}_{\partial \Omega}(x)$ the outer normal to $\partial \Omega$ at $x \in \partial \Omega$ and by $\hat{n}_{\partial D}(y)$ the outer normal to $\partial D$ at $y \in \partial D$. Let $h$ be any positive quantity and $\Omega_{h, v}=\Omega-h v$. We observe that:

(a) if $x \notin \partial D \backslash \bar{\Omega}$ there exists $\bar{h}_{x}>0$ such that $x \notin \partial D \cap \Omega_{h, v}$ for any $h \in\left(0, h_{x}\right)$;

(b) if $x \in \partial D \cap \Omega$ there exists $\bar{h}_{x}>0$ such that $x \in \partial D \cap \Omega_{h, v}$ for any $h \in\left(0, h_{x}\right)$;

(c) if $x \in \partial D \cap \partial \Omega \cap P_{v}^{-}$there exists $\bar{h}_{x}>0$ such that $x \in \partial D \cap \Omega_{h, v}$ for any $h \in\left(0, h_{x}\right)$;

(d) if $x \in \partial D \cap \partial \Omega \cap P_{v}^{+}$and $\hat{n}_{\partial \Omega}(x)=\hat{n}_{\partial D}(x)$ there exists $\bar{h}_{x}>0$ such that $x \notin$ $\partial D \cap \Omega_{x, v}$ for any $h \in\left(0, h_{x}\right)$

(e) $\mathcal{H}^{1}\left(\left\{x \in \partial D \cap \partial \Omega: \hat{n}_{\partial \Omega}(x) \neq \hat{n}_{\partial D}(x)\right\}\right)=0$. 
We denote by $\chi_{h}^{+}: T_{v} \rightarrow[0,1]$ the characteristic function of $\Pi_{v}\left(\Omega_{h, v} \cap \partial D \cap P_{v}^{+}\right)$and by $\chi_{h}^{-}: T_{v} \rightarrow[0,1]$ the characteristic function of $\Pi_{v}\left(\Omega_{h, v} \cap \partial D \cap P_{v}^{-}\right)$. A straightforward consequence of (a)-(d) is that

$$
\begin{array}{ll}
\lim _{h \rightarrow 0^{+}} \chi_{h}^{+}=\chi_{\Pi_{v}\left(A \cap P_{v}^{+}\right)} & \text {a.e. on } T_{v}, \\
\lim _{h \rightarrow 0^{+}} \chi_{h}^{-}=\chi_{\Pi_{v}\left((A \cup G) \cap P_{v}^{-}\right)} & \text {a.e. on } T_{v} .
\end{array}
$$

If we set

$$
f(h)=L\left(\Pi_{v}\left(\Omega_{h, v} \cap \partial D \cap P_{v}^{+}\right)\right), \quad g(h)=L\left(\Pi_{v}\left(\Omega_{h, v} \cap \partial D \cap P_{v}^{-}\right)\right),
$$

then using the Fubini theorem and the optimality of $D$ with respect to $\Omega$ we obtain

$$
\int_{0}^{h}(f(s)-g(s)) d s=|\Omega \backslash D|-\left|\Omega_{h, v} \backslash D\right| \leq 0 .
$$

In the limit as $h$ goes to 0 this yields $f\left(0^{+}\right) \leq g\left(0^{+}\right)$and the claim easily follows.

Lemma 4.2. Let $v$ be any direction in $\mathbb{R}^{2}$. Then

$$
L\left(\Pi_{v}\left(B \cap P_{v}^{+}\right)\right) \leq L\left(\Pi_{v}\left(B \cap P_{v}^{-}\right)\right)+L\left(\Pi_{v}\left(G \cap P_{v}^{-}\right)\right) .
$$

Proof. The claim easily follows from the previous lemma on interchanging $P_{v}^{+}$and $P_{v}^{-}$, once we observe that

$$
L\left(\Pi_{v}\left(A \cap P_{v}^{ \pm}\right)\right)+L\left(\Pi_{v}\left(G \cap P_{v}^{ \pm}\right)\right)+L\left(\Pi_{v}\left(B \cap P_{v}^{ \pm}\right)\right)=2 R .
$$

The following lemma gives a relation between arclengths.

Lemma 4.3. For any $i \in \mathfrak{I}$ and $k \in \mathfrak{K}$,

$$
L\left(\alpha_{i}\right) \leq \sum_{j \neq i} L\left(\alpha_{j}\right)+L(G), \quad L\left(\beta_{k}\right) \leq \sum_{j \neq k} L\left(\beta_{j}\right)+L(G) .
$$

Proof. First of all we observe that $\alpha_{i}$ and $\beta_{k}$ cannot be longer than $\pi R$. Indeed, if for instance $L\left(\alpha_{i}\right)>\pi R$, then there exists a direction $v$ such that $\partial D \cap P_{v}^{+} \subset \alpha_{i}$ and from Lemma 4.1 one easily gets $B \cap P_{v}^{-}=\emptyset$ and therefore $|\Omega|>|D|$, contrary to the definition of $D$.

We then apply Lemma 4.1 to $\alpha_{i}$ choosing the direction $v$ to be orthogonal to the chord corresponding to $\alpha_{i}$. Similarly, we apply Lemma 4.2 to $\beta_{k}$ choosing $v$ orthogonal to the chord corresponding to $\beta_{k}$. The claim of the lemma then easily follows from the trivial observation that among all the rectifiable sets $\gamma \subset \partial D \cap P_{v}^{+}$of fixed measure, $L\left(\Pi_{v}(\gamma)\right)$ reaches its maximum if and only if $\gamma$ is an arc whose chord is orthogonal to $\nu$.

Lemma 4.4. For any $i \in \mathfrak{I}$ and $k \in \mathfrak{K}, L\left(\alpha_{i}\right)+L\left(\beta_{k}\right) \leq \pi R$. 
Proof. Assume that

$$
L\left(\alpha_{i}\right)+L\left(\beta_{k}\right)>\pi R .
$$

As already observed in Lemma 4.3 we have $L\left(\alpha_{i}\right) \leq \pi R$ and $L\left(\beta_{k}\right) \leq \pi R$, therefore there exists a direction $v$ such that $\alpha_{i} \subset P_{v}^{+}$and $\beta_{k} \subset P_{v}^{-}$. Denote by $\varpi_{1}$ and $\varpi_{2}$ the two connected components (one possibly empty) of $\left(\partial D \cap P_{v}^{-}\right) \backslash \beta_{k}$. Then we set $\bar{\theta}=R^{-1} L\left(\alpha_{i}\right), \theta_{1}=R^{-1} L\left(\varpi_{1}\right)$ and $\theta_{2}=R^{-1} L\left(\varpi_{2}\right)$. Inequality 4.3 yields $\theta_{1}+\theta_{2}<\bar{\theta}$ and using Lemma 4.1 we get

$$
\begin{aligned}
R\left(1-\cos \left(\theta_{1}+\theta_{2}\right)\right) & <R(1-\cos \bar{\theta}) \leq L\left(\Pi_{v}\left(\alpha_{i}\right)\right) \\
& \leq L\left(\Pi_{v}\left(A \cap P_{v}^{+}\right)\right) \leq 2 R-L\left(\Pi_{v}\left(B \cap P_{v}^{-}\right)\right) \\
& \leq 2 R-L\left(\Pi_{v}\left(\beta_{k}\right)\right)=L\left(\Pi_{v}\left(\varpi_{1}\right)\right)+L\left(\Pi_{v}\left(\varpi_{2}\right)\right) \\
& =R\left(1-\cos \theta_{1}\right)+R\left(1-\cos \theta_{2}\right), \\
& =R\left(1-\cos \theta_{1} \cos \theta_{2}+\left(1-\cos \theta_{1}\right)\left(1-\cos \theta_{2}\right)\right) \\
& \leq R\left(1-\cos \theta_{1} \cos \theta_{2}+\sqrt{\left(1-\cos ^{2} \theta_{1}\right)\left(1-\cos ^{2} \theta_{2}\right)}\right) \\
& =R\left(1-\cos \left(\theta_{1}+\theta_{2}\right)\right),
\end{aligned}
$$

which is a contradiction. We have used the fact that

$$
\theta_{1}, \theta_{2} \geq 0 \text { and } \theta_{1}+\theta_{2} \leq \pi
$$

which enforces $\cos \theta_{1}+\cos \theta_{2} \geq 0$ and hence

$$
\sqrt{\left(1+\cos \theta_{1}\right)\left(1+\cos \theta_{2}\right)} \geq \sqrt{\left(1-\cos \theta_{1}\right)\left(1-\cos \theta_{2}\right)} .
$$

Finally the following lemma will be of importance when applying the pseudo-circular symmetrization.

\section{Lemma 4.5. Let}

$$
I=\max \left\{\max _{k} L\left(\beta_{k}\right), \frac{1}{2} \sum_{k} L\left(\beta_{k}\right)\right\}, \quad J=\max \left\{\max _{i} L\left(\alpha_{i}\right), \frac{1}{2} \sum_{i} L\left(\alpha_{i}\right)\right\} .
$$

Then $I+J \leq \pi R$.

Proof. In view of Lemma 4.4 and the trivial inequality

$$
\frac{1}{2} \sum_{j} L\left(\alpha_{j}\right)+\frac{1}{2} \sum_{j} L\left(\beta_{j}\right) \leq \pi R
$$

it is enough to prove that for any $k \in \mathfrak{K}$,

$$
L\left(\beta_{k}\right)+\frac{1}{2} \sum_{i} L\left(\alpha_{i}\right) \leq \pi R
$$

Assume that

$$
2 L\left(\beta_{k}\right)+\sum_{i} L\left(\alpha_{i}\right)>2 \pi R
$$


Using the trivial equality

$$
\sum_{j} L\left(\alpha_{j}\right)+\sum_{j} L\left(\beta_{j}\right)+L(G)=2 \pi R
$$

we get

$$
L\left(\beta_{k}\right)>\sum_{j \neq k} L\left(\beta_{j}\right)+L(G)
$$

which contradicts Lemma 4.3 . In a similar way one can show that for any $i \in \mathfrak{I}$,

$$
L\left(\alpha_{i}\right)+\frac{1}{2} \sum_{k} L\left(\beta_{k}\right) \leq \pi R .
$$

\section{Proof of the main result}

We are ready to prove our main result by presenting a reduction algorithm. For the reader's convenience we divide the proof into three steps. At each step the procedure preserves the Fraenkel asymmetry index while not increasing the isoperimetric deficit. In Step 1 we reduce a given bounded convex set to a new set (not necessarily convex) having two orthogonal axes of symmetry. In Step 2 we reshape the set to obtain a set with boundary consisting only of circular arcs, but the set is not necessarily convex or smooth. Finally in Step 3 we show that among all the sets of fixed asymmetry index given in Step 2, the optimal one (having the smallest possible isoperimetric deficit) is the unique (up to similarity) smooth set. Incidentally, such a set is convex.

\subsection{Step 1: Reduction to a 2-symmetric set}

Let us show how to reduce any given convex set $\Omega$ to a set having two orthogonal axes of symmetry, exactly the same measure and Fraenkel asymmetry index of $\Omega$ but a perimeter not greater than $P(\Omega)$.

We consider two directions $v_{1}$ and $\nu_{2}$ and the two half-lines originating from $O$ and containing them which split the plane in two angles that we denote by $\mathcal{A}_{1}$ and $\mathcal{A}_{2}$. We fix $v_{1}$ and $v_{2}$ according to the occurrence of one of the following cases:

(A1) for all $i \in \mathfrak{I}, L\left(\alpha_{i}\right) \leq \sum_{j \neq i} L\left(\alpha_{j}\right)$;

(A2) there exists $\underline{i} \in \mathfrak{I}$ such that $L\left(\alpha_{\underline{i}}\right)>\sum_{j \neq \underline{i}} L\left(\alpha_{j}\right)$.

In the first case we fix $\nu_{1}$ and $v_{2}$ such that $\alpha_{1} \subset \mathcal{A}_{1}, \alpha_{2} \subset \mathcal{A}_{2}$ and $L\left(A \cap \mathcal{A}_{1}\right)=L\left(A \cap \mathcal{A}_{2}\right)$. In the second case we choose $v_{1}$ and $\nu_{2}$ such that $\alpha_{i}=A \cap \mathcal{A}_{1}$.

In a similar way if $\mu_{1}$ and $\mu_{2}$ are two directions and the two half-lines originating from the origin and containing them split the plane in two angles denoted by $\mathcal{B}_{1}$ and $\mathcal{B}_{2}$, we can always fix $\mu_{1}$ and $\mu_{2}$ according to the occurrence of one of the following cases:

(B1) for all $k \in \mathfrak{K}, L\left(\beta_{k}\right) \leq \sum_{j \neq k} L\left(\beta_{j}\right)$;

(B2) there exists $\hat{k} \in \mathfrak{K}$ such that $L\left(\beta_{\hat{k}}\right)>\sum_{j \neq \hat{k}} L\left(\beta_{j}\right)$. 
In the first case we fix $\mu_{1}$ and $\mu_{2}$ such that $\beta_{1} \subset \mathcal{B}_{1}, \beta_{2} \subset \mathcal{B}_{2}$ and $L\left(B \cap \mathcal{B}_{1}\right)=L\left(B \cap \mathcal{B}_{2}\right)$. In the second case we choose $\mu_{1}$ and $\mu_{2}$ such that $\beta_{\hat{k}}=B \cap \mathcal{B}_{1}$.

Next we consider $\Omega^{+}=D \cup \Omega$ and $\Omega^{-}=D \cap \Omega$ (see Figures 4 (b) and 4 (c)) and their pseudo-circular symmetrizations that we denote $\Omega^{v_{1} \nu_{2}}$ and $\Omega_{\mu_{1} \mu_{2}}$ (see Figures $4 \mathrm{~d}$ ) and $4(\mathrm{e}))$.

Remark 5.1. In the notation of Section 3, suppose that the coordinate axes are chosen in such a way that $\nu_{1}$ and $\nu_{2}$ correspond in polar coordinates to $\theta=0$ and $\theta=\bar{\theta}$. The radial function of the set $\Omega^{+}$is $\max \{\rho(\theta), R\}$, and from the definition of $v_{1}$ and $v_{2}$ we notice that $\max \{\rho(\theta), R\}$ belongs to both $\Lambda(0, \bar{\theta})$ and $\Lambda(\bar{\theta}, 2 \pi)$.

Similarly, the radial function of the set $\Omega^{-}$is $\min \{\rho(\theta), R\}$. In that case we assume that $\mu_{1}$ and $\mu_{2}$ correspond in polar coordinates to 0 and $\bar{\theta}$. Then the definition of $\mu_{1}$ and $\mu_{2}$ implies that $\min \{\rho(\theta), R\}$ belongs to both $\Lambda(0, \bar{\theta})$ and $\Lambda(\bar{\theta}, 2 \pi)$.

By definition the set $\Omega^{v_{1} v_{2}}$ is symmetric with respect to the axis in the direction $v_{1}+v_{2}$, while $\Omega^{\mu_{1} \mu_{2}}$ is symmetric with respect to the axis in the direction $\mu_{1}+\mu_{2}$. We can rotate the two sets around the point $O$ until their symmetry axes happen to be orthogonal (see Figures $4(d)$ and $4(e))$. From now on we use these orthogonal axes as a reference system $(\xi, \eta)$.

We merge the sets $\Omega^{\nu_{1} \nu_{2}}$ and $\Omega_{\mu_{1} \mu_{2}}$ into the set $\Omega_{0}=\left(\Omega^{\nu_{1} \nu_{2}} \backslash D\right) \cup \Omega_{\mu_{1} \mu_{2}}$ (see Figure 4(f)). The following result holds.

Lemma 5.1. $\Omega_{0}$ has the following properties:

(1) $\Omega_{0}$ is starshaped;

(2) $\left|\Omega_{0}\right|=|\Omega|$;

(3) $\left|\Omega_{0} \backslash D\right|=|\Omega \backslash D|$;

(4) $\left|\partial \Omega_{0}\right| \leq|\partial \Omega|$.

Proof. We observe that by definition

$$
L\left(A \cap \mathcal{A}_{1}\right) \geq L\left(A \cap \mathcal{A}_{2}\right) \quad \text { and } \quad L\left(B \cap \mathcal{B}_{1}\right) \geq L\left(B \cap \mathcal{B}_{2}\right) .
$$

Moreover by Lemma 4.5 we have

$$
L\left(A \cap \mathcal{A}_{1}\right)+L\left(B \cap \mathcal{B}_{1}\right) \leq \pi R .
$$

This implies that $\Omega_{0}$ is starshaped.

Properties (2)-(3) rely on the following observation: $\Omega^{\nu_{1} \nu_{2}}$ and $\Omega_{\mu_{1} \mu_{2}}$ are the sets whose radial functions in polar coordinates are rearrangements of the radial functions of $\Omega^{+}$and $\Omega^{-}$. Therefore the parametrization $\rho_{0}(\theta)$ of $\partial \Omega_{0}$ in polar coordinates is equimeasurable with $\rho(\theta)$, i.e.

$$
|\{\rho>t\}|=\left|\left\{\rho_{0}>t\right\}\right| \quad \forall t \geq 0 .
$$

Finally by Remark 5.1 and Lemma 3.1. $\left|\partial \Omega^{\nu_{1} \nu_{2}}\right| \leq\left|\partial \Omega^{+}\right|$and $\left|\partial \Omega_{\mu_{1} \mu_{2}}\right| \leq\left|\partial \Omega^{-}\right|$and property (4) follows immediately.

After the above operation, the set $\Omega_{0}$ is such that the part of the boundary of $D$ given by $\partial D \backslash \partial \Omega_{0}$ consists of at most four arcs which we denote by $a_{1}, a_{2}, b_{1}, b_{2}$ so that 
$L\left(a_{1}\right)=L\left(A \cap \mathcal{A}_{1}\right), L\left(a_{2}\right)=L\left(A \cap \mathcal{A}_{2}\right), L\left(b_{1}\right)=L\left(B \cap \mathcal{B}_{1}\right)$ and $L\left(b_{2}\right)=L\left(B \cap \mathcal{B}_{2}\right)$. Without loss of generality, we assume that $a_{1}$ belongs to the half-plane $\xi \geq 0$. If we neglect the trivial case $\Omega=D$ then $a_{1} \neq \varnothing$ and $b_{1} \neq \emptyset$. One of the following four cases certainly occurs:

(o1) $a_{2} \neq \emptyset$ and $b_{2} \neq \emptyset$;

(o2) $a_{2}=\emptyset$ and $b_{2} \neq \emptyset$;

(o3) $a_{2}=\emptyset$ and $b_{2}=\emptyset$;

(o4) $a_{2} \neq \emptyset$ and $b_{2}=\emptyset$.

In any case

$$
\begin{array}{ll}
a_{1} \cup a_{2}=\partial D \cap \Omega_{0}, & L\left(a_{1}\right) \geq L\left(a_{2}\right), \\
b_{1} \cup b_{2}=\partial D \backslash \bar{\Omega}_{0}, & L\left(b_{1}\right) \geq L\left(b_{2}\right) .
\end{array}
$$

Each of the arcs $a_{1}, a_{2}$ is symmetric with respect to the $\xi$-axis, while $b_{1}$ and $b_{2}$ are symmetric with respect to the $\eta$-axis. Let $\bar{d}=R \sin \left(L\left(a_{1}\right) / 2 R\right)$ and let $l_{1}$ and $l_{2}$ be two lines orthogonal to $\eta$ and having the same distance $\bar{d}$ from the origin $O$ (see Figure 4 (f)). We denote by $\Sigma_{\text {in }}$ the open subset of $\mathbb{R}^{2}$ between these two lines and by $\Sigma_{\text {out }}$ the open set $\mathbb{R}^{2} \backslash \bar{\Sigma}_{\text {in }}$. Moreover we denote by $\mathcal{C}$ the smallest angle with vertex at the origin and containing $a_{1}$, and by $-\mathcal{C}$ its symmetric image with respect to the $\eta$-axis. Now we can state the following result.

Lemma 5.2. The lines $l_{1}$ and $l_{2}$ intersect $\partial D$ in four points which belong to $\partial \Omega_{0}$. Moreover the sets $\Omega_{0} \backslash \bar{D}$ and $D \backslash \bar{\Omega}_{0}$ are subsets of $\Sigma_{\text {in }}$ and $\Sigma_{\text {out }}$ respectively.

Proof. It immediately follows from the definition of $\bar{d}$ that

$$
a_{i} \subset \Sigma_{\text {in }}, \quad i=1,2 .
$$

According to inequality (5.1) we have

$$
L\left(a_{1}\right)+L\left(b_{1}\right) \leq \pi R .
$$

We deduce that

$$
b_{i} \subset \Sigma_{\text {out }}, \quad i=1,2,
$$

which also proves the first assertion.

In order to prove that $\Omega_{0} \backslash \bar{D} \subset \Sigma_{\text {in }}$ we show that the radial function of $\Omega_{0}$ restricted to the angles $\mathcal{C}$ and $-\mathcal{C}$ is bounded from above by the radial function of $l_{1} \cup l_{2}$ restricted to the same angles. By using the symmetry of the set $\Omega_{0}$ it will be enough to prove an inequality involving the level sets of these functions.

Since $L\left(b_{1}\right)=L\left(B \cap \mathcal{B}_{1}\right) \geq \max _{k} L\left(\beta_{k}\right)$ and the distance from 0 to the chord of $b_{1}$ is not smaller than $\bar{d}$, it immediately follows that $D_{\bar{d}}(0) \subset \Omega$. It follows that (see [29]) the radial function $\rho$ of $\Omega$ is a Lipschitz function such that

$$
\left|\frac{\rho\left(\theta_{1}\right)-\rho\left(\theta_{2}\right)}{\theta_{1}-\theta_{2}}\right| \leq \frac{\hat{\rho}}{\bar{d}} \sqrt{\hat{\rho}^{2}-\bar{d}^{2}}
$$




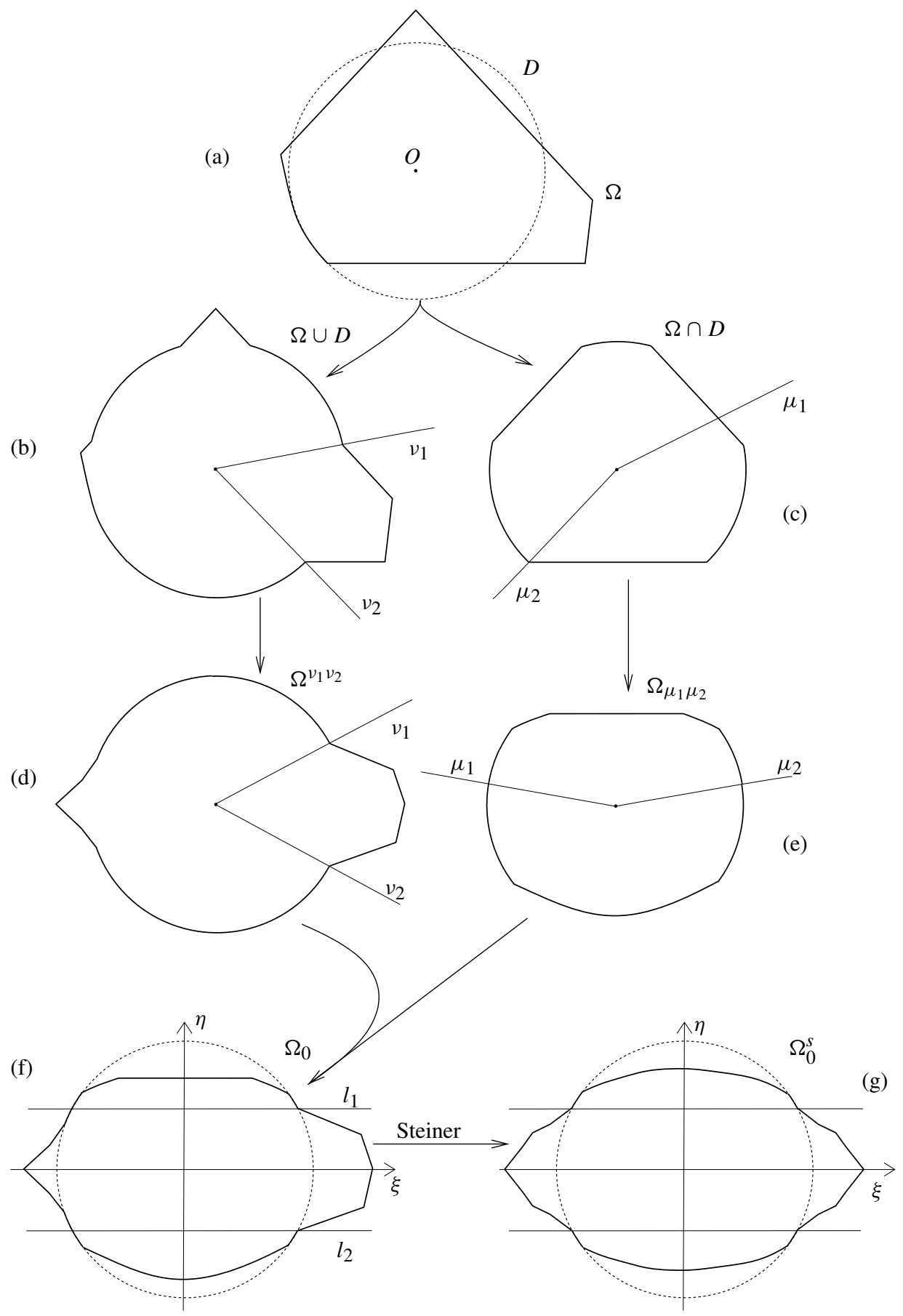

Fig. 4. The symmetrization procedure transforming $\Omega$ into $\Omega_{0}^{s}$. 
where $\hat{\rho}=\max \left\{\rho\left(\theta_{1}\right), \rho\left(\theta_{2}\right)\right\}$. Since it makes sense to consider the right and left derivatives of $\rho$, denoted $\rho^{\prime}\left(\theta^{+}\right)$and $\rho^{\prime}\left(\theta^{-}\right)$, by passing to the limit when $\theta_{2} \rightarrow \theta_{1}$ we get

$$
\left|\rho^{\prime}\left(\theta^{ \pm}\right)\right| \leq \frac{\rho(\theta)}{\bar{d}} \sqrt{\rho(\theta)^{2}-\bar{d}^{2}} \quad \forall \theta \in[0,2 \pi] .
$$

Now, we consider the restrictions $\rho_{1}$ and $\rho_{2}$ of the function $\rho$ to the angles $\mathcal{A}_{1}$ and $\mathcal{A}_{2}$. By the definitions of $\mathcal{A}_{1}$ and $\mathcal{A}_{2}$ for $i=1,2$ we have

$$
\#\left\{\rho_{i}=t\right\} \geq 2 \quad \text { for } R<t<\max \rho_{i},
$$

therefore the coarea formula yields

$$
\begin{aligned}
\left|\left\{\rho_{i}>t\right\}\right| & \leq\left|\left\{\rho_{i}>R\right\}\right|-\int_{R}^{t} d s \int_{\rho_{i}=s} \frac{1}{\left|\rho_{i}^{\prime}\right|} d \mathcal{H}^{o} \leq \frac{L\left(a_{i}\right)}{R}-\int_{R}^{t} \frac{2 \bar{d}}{s \sqrt{s^{2}-\bar{d}^{2}}} d s \\
& \leq \frac{L\left(a_{1}\right)}{R}-2 \arcsin \frac{\bar{d}}{R}+2 \arcsin \frac{\bar{d}}{t}=2 \arcsin \frac{\bar{d}}{t} .
\end{aligned}
$$

On the other hand, if $\varphi=L\left(a_{1}\right) /(2 R)$, the functions

$$
\begin{array}{ll}
r_{1}(\theta)=\frac{\bar{d}}{|\sin \theta|}, & \theta \in(0, \varphi) \cup(2 \pi-\varphi, 2 \pi), \\
r_{2}(\theta)=\frac{\bar{d}}{|\sin \theta|}, & \theta \in(\pi-\varphi, \pi+\varphi),
\end{array}
$$

have distribution functions

$$
\begin{aligned}
& m_{1}(t)=\left|\left\{\theta \in(0, \varphi) \cup(2 \pi-\varphi, 2 \pi): r_{1}(\theta) \geq t\right\}\right|=2 \arcsin \frac{\bar{d}}{t}, \\
& m_{2}(t)=\left|\left\{\theta \in(\pi-\varphi, \pi+\varphi): r_{2}(\theta) \geq t\right\}\right|=2 \arcsin \frac{\bar{d}}{t} .
\end{aligned}
$$

Because of (5.2) and (5.3) the function $\rho_{1}^{\sharp}(\theta)$ describes in polar coordinates a curve which in $\mathcal{C}$ coincides with $\partial \Omega_{0}$ while the function $\max \left\{\rho_{2}^{\sharp}(\theta-\pi), R\right\}$ describes a curve which in $-\mathcal{C}$ coincides with $\partial \Omega_{0}$. Since $r(\theta)=\bar{d}\left|(\sin \theta)^{-1}\right|$ is the radial function of the two lines $l_{1}$ and $l_{2}$, by comparing inequalities (5.5) with (5.6) and (5.7) we conclude that $\Omega_{0} \backslash \bar{D} \subset \Sigma_{\text {in }}$. The proof of $D \backslash \bar{\Omega}_{0} \subset \Sigma_{\text {out }}$ can be obtained in a similar way.

We denote by $\Omega_{0}^{s}$ the Steiner symmetrization of $\Omega_{0}$ with respect to both axes $\xi$ and $\eta$ (see Figure $4 \mathrm{~g}$ )). Lemmas $5.1 \sqrt{5.2}$ both hold on replacing $\Omega_{0}$ by $\Omega_{0}^{s}$ with the same lines $l_{1}$ and $l_{2}$ defined above. The set $\Omega_{0}^{s}$ is the desired 2 -symmetric set, which concludes this step.

Remark 5.2. We observe that, by slightly modifying the last part of the proof of Lemma 5.2 , it is possible to prove a regularity property of the boundary of $\Omega_{0}$, namely that $\partial \Omega_{0} \cap \Sigma_{\text {in }}$ can be locally represented as the graph of a Lipschitz function with respect to the $\eta$-axis. Similarly $\partial \Omega_{0} \cap \Sigma_{\text {out }}$ can be locally represented as the graph of a Lipschitz 
function with respect to the $\xi$-axis. By the property of the Steiner symmetrization we deduce that $\partial \Omega_{0}^{s} \cap \Sigma_{\text {in }}$ can be locally represented as the graph of a Lipschitz function with respect to the $\eta$-axis, while $\partial \Omega_{0}^{s} \cap \Sigma_{\text {out }}$ can be locally represented as the graph of a Lipschitz function with respect to the $\xi$-axis.

Remark 5.3. We came up with the set $\Omega_{0}^{s}$ only after much effort since, as we indicated at the beginning of the section, we were looking for an algorithm which leaves the asymmetry index unchanged during the process of symmetrization. Indeed, by using Lemmas 5.1 and 5.2 it is possible to show that the disk $D$ is still the optimal one, i.e.

$$
\alpha\left(\Omega_{0}^{s}\right)=\frac{\left|\Omega_{0}^{s} \backslash D\right|}{\left|\Omega_{0}^{s}\right|} .
$$

The importance of the last condition is evident. Nevertheless to get through the next step we do not need it.

\subsection{Step 2: Reduction to a 2-symmetric set with the boundary made of arcs}

Definition 5.1 (The family $\mathfrak{Z}$ ). We say that $E$ belongs to the family $\mathfrak{Z}\left(\alpha_{0}, m\right)$ (for some $m \geq 0$ and $0 \leq \alpha_{0}<1$ ) if up to a rototranslation:

(a1) $E$ is starshaped with respect to $O$;

(a2) $E$ has two orthogonal axes of symmetry both passing through $O$;

(a3) $|E|=m$;

(a4) $|E \backslash D|=\alpha_{0}|E|$, where $D$ is the disk having center at $O$ and radius $(|E| / \pi)^{1 / 2}$;

(a5) in the notation of the previous subsection, there exist two lines $l_{1}$ and $l_{2}$ orthogonal to the $\eta$-axis and having the same distance from $O$ such that they intersect $\partial D$ in four points which also belong to $\partial E$; moreover, $E \backslash D$ and $D \backslash E$ are subsets of $\Sigma_{\text {in }}$ and $\Sigma_{\text {out }}$ respectively.

Remark 5.4. Because of the hypotheses (a1)-(a5), it immediately follows that

$$
\alpha(E)=\frac{|E \backslash D|}{|E|}, \quad E \in \mathfrak{Z}\left(\alpha_{0}, m\right) .
$$

The set $\Omega_{0}^{s}$ obtained above in Step 1 belongs to $\mathfrak{Z}(\alpha(\Omega),|\Omega|)$ when the lines $l_{1}$ and $l_{2}$ are defined as in the previous subsection, and $\partial D \backslash \partial \Omega_{0}^{s}$ is made of four $\operatorname{arcs} d_{1}, d_{2}, e_{1}, e_{2}$ such that

$$
\begin{array}{ll}
L\left(d_{1}\right)=L\left(d_{2}\right), & L\left(e_{1}\right)=L\left(e_{2}\right), \\
d_{1} \cup d_{2} \subset \bar{\Sigma}_{\text {in }}, & e_{1} \cup e_{2} \subset \bar{\Sigma}_{\text {out }} .
\end{array}
$$

Let us consider the four points given by the intersection of $l_{1}$ and $l_{2}$ with $\partial D$. They are vertices of a rectangle and we denote them $P_{i}, i=1, \ldots, 4$, assuming that starting from the upper left corner they are placed in the clockwise order. It is trivial to check that $P_{i} \in \partial \Omega_{0}^{s}$ for all $1 \leq i \leq 4$. We look for a set belonging to $\mathfrak{Z}(\alpha(\Omega),|\Omega|)$, having minimum perimeter, with the further constraint that in (a5) of Definition 5.1 the lines $l_{1}$ 
and $l_{2}$ are fixed. This implies in particular that $P_{1}, P_{2}, P_{3}, P_{4}$ belong to its boundary. By symmetry the problem can be solved by finding the optimal shape of the boundary, namely by looking for two curves $g_{1}$ and $g_{2}$ having the smallest possible length and satisfying the following properties:

(b1) $g_{1}$ connects $P_{1}$ and $P_{2}$;

(b2) $g_{1} \subset \bar{\Sigma}_{\text {out }} \cap D$;

(b3) the region between $\partial D \cap \Sigma_{\text {out }}$ and $g_{1}$ has measure $\frac{1}{2} \alpha(\Omega)|\Omega|$;

(b4) $g_{2}$ connects $P_{2}$ and $P_{3}$;

(b5) $g_{2} \subset \bar{\Sigma}_{\text {in }} \backslash D$;

(b6) the region between $\partial D \cap \Sigma_{\text {in }}$ and $g_{2}$ has measure $\frac{1}{2} \alpha(\Omega)|\Omega|$.

The set $\Omega^{a} \in \mathfrak{Z}(\alpha(\Omega),|\Omega|)$ having $g_{1}$ and $g_{2}$ as part of the boundary (the rest of the boundary can be deduced using symmetry) has the desired properties. A simple variational argument shows that $g_{1}$ has to be a circular arc. As regards $g_{2}$, the same argument can be applied if $\alpha(\Omega)$ is small enough, namely,

$$
\alpha(\Omega) \leq \frac{1}{|\Omega|}\left(\pi d^{2}+2 d \sqrt{\frac{|\Omega|}{\pi}-d^{2}}-2\left(\frac{|\Omega|}{\pi}\right)^{1 / 2} \arcsin \frac{d}{r}\right) .
$$

If condition (5.8) is not satisfied, we can find $g_{2}$ using an argument contained in [25, Th. 3.32]. Indeed, $\Omega^{a} \cap \Sigma_{\text {in }}$ is the convex hull of two balls of radius $d / 2$. In other words $g_{2}$ is the only $C^{1}$ curve given by the union of a circular arc (more precisely half circumference) with two segments $k_{1}$ and $k_{2}$ of equal length, lying on $l_{1}$ and $l_{2}$.

\subsection{Step 3: Reduction to a smooth convex set}

In this subsection we prove that among all the sets in $\mathfrak{Z}(\alpha(\Omega), \pi)$, up to similarity, the set $S_{\alpha(\Omega)}$ achieves the minimum isoperimetric deficit.

We begin by proving that, for any $m \geq 0$ and $0 \leq \alpha_{0}<1$, there exists a set, the optimal set, having minimum perimeter among all the sets belonging to $\mathfrak{Z}\left(\alpha_{0}, m\right)$.

If we denote by $\mathfrak{Z}^{a}\left(\alpha_{0}, m\right)$ the family $\left\{E \in \mathfrak{Z}\left(\alpha_{0}, m\right): E=E^{a}\right\}$, a trivial consequence of the previous subsections is that we achieve our goal if we can prove that there exists a set having minimum perimeter in $\mathfrak{Z}^{a}\left(\alpha_{0}, m\right)$.

The symmetry allows us to restrict our analysis to the part of the $(\xi, \eta)$-plane with $\xi, \eta \geq 0$; we observe that any element in $\mathfrak{Z}^{a}\left(\alpha_{0}, m\right)$ is uniquely determined by the point $P=\left(\xi_{0}, \sqrt{m / \pi-\xi_{0}^{2}}\right)$ of intersection of its boundary with $\partial D$. A straightforward calculation shows that $\mathfrak{Z}^{a}\left(\alpha_{0}, m\right)$ is a one-parameter family of sets. Indeed, if $0<\bar{\xi}<$ $(m / \pi)^{1 / 2}$ is chosen such that

$$
\alpha_{0}=\frac{1}{m}\left(2\left(\frac{m}{\pi}\right)^{1 / 2} \arcsin \left[\bar{\xi}\left(\frac{\pi}{m}\right)^{1 / 2}\right]-2 \bar{\xi} \sqrt{\frac{m}{\pi}-\bar{\xi}^{2}}\right),
$$


for any given $\bar{\xi} \leq \xi_{0}<(m / \pi)^{1 / 2}$ there exists only one set $E_{\xi_{0}} \in \mathfrak{Z}^{a}\left(\alpha_{0}, m\right)$ whose boundary passes through the point $P \equiv\left(\xi_{0}, \sqrt{m / \pi-\xi_{0}^{2}}\right)$ and vice versa. Since the isoperimetric deficit $\Delta P\left(E_{\xi_{0}}\right)$ changes continuously with $\xi_{0}$ and

$$
\lim _{\xi_{0} \rightarrow(m / \pi)^{1 / 2}} \Delta P\left(E_{\xi_{0}}\right)=+\infty
$$

we can conclude that, among the sets belonging to $\mathfrak{Z}^{a}\left(\alpha_{0}, m\right)$ there exists at least one having minimum perimeter.

Now it is not difficult to see that $S_{\alpha_{0}}$ belongs to $\mathfrak{Z}^{a}\left(\alpha_{0}, \pi\right)$ and that, up to similarity, it is the unique smooth set (with $C^{1}$ boundary) in $\mathfrak{Z}^{a}\left(\alpha_{0}, m\right)$. Our goal is to prove that this set is the optimal set we are looking for. All we have to do is to prove that a non-smooth set cannot be the optimal one.

A given set $E \in \mathfrak{Z}^{a}\left(\alpha_{0}, m\right)$ is smooth except at worst at the point $P$ of intersection with the boundary of the optimal disk $D$ (see Figure 5 (a)-(b)). When the boundary of $E$ is non-smooth, zooming out around the point $P$, the boundary will look like Figure 5 (c). By using the same notation in Figures 5(a)-(b) and in Figure 5(c), $\overline{A P}$ and $\overline{P B}$ represent pieces of the boundary of $E$. Due to the zoom around the point $P$, in Figure 5 (c), all the arcs are schematically represented and treated as segments. Here $\overline{P C}$ represents a piece of $\partial D$.

We want to show that if two points $Q^{\prime}$ and $Q^{\prime \prime}$ are chosen in an appropriate way then in the boundary of $E$ we can replace the polygonal chain $\overline{A P B}$ by $\overline{A Q^{\prime} Q^{\prime \prime} B}$. The new set will have a shorter perimeter but will still belong to $\mathfrak{Z}\left(\alpha_{0}, m\right)$. For this purpose, let $Q^{\prime}$ and $Q^{\prime \prime}$ be two points such that:

- $\overline{Q^{\prime} Q^{\prime \prime}}$ is parallel to $\overline{A B}$;

- $\overline{Q^{\prime} Q^{\prime \prime}}$ intersects $\overline{P C}$ in a point denoted by $D$;

- the area of the trapezoid $A Q^{\prime} D C$ is equal to the area of $A P C$;

- the area of the trapezoid $C D Q^{\prime \prime} B$ is equal to the area of $C P B$.

A straightforward but tedious computation yields

$$
(|\overline{A P}|+|\overline{P B}|)-\left(\left|\overline{A Q^{\prime}}\right|+\left|\overline{Q^{\prime} Q^{\prime \prime}}\right|+\left|\overline{Q^{\prime \prime} B}\right|\right)=K|\overline{P D}|+o(|\overline{P D}|)
$$

where $K=(|\overline{A P}|+|\overline{P B}|+|\overline{A B}|) /|\overline{P C}|>0$ is a constant that does not depend on $Q^{\prime}, Q^{\prime \prime}$ and $D$. Therefore if in addition to the previous hypotheses we take $Q^{\prime}$ and $Q^{\prime \prime}$ such that $|\overline{P D}|$ is small enough, the picture in Figure 5 (c) represents a way to replace the polygonal chain $\overline{A P B}$ by $\overline{A Q^{\prime} Q^{\prime \prime} B}$ shortening the perimeter of $E$ without changing $|E|$ or $|E \backslash D|$. We conclude that a non-smooth set cannot be optimal.

\subsection{The equality case}

To complete the proof of Theorem 2.1 we need to characterize the sets which achieve equality in 2.4. Let $\Omega$ be such a set. We want to prove that $\Omega \in \mathfrak{S}$. We shall not consider the trivial case $\alpha(\Omega)=0$. 
(a)

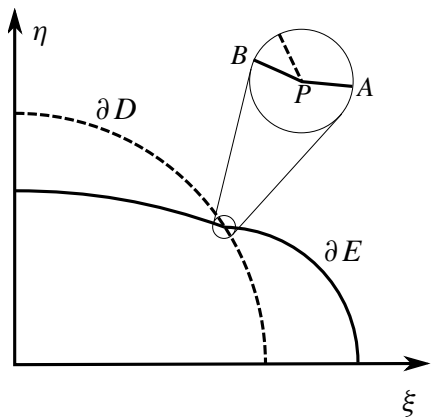

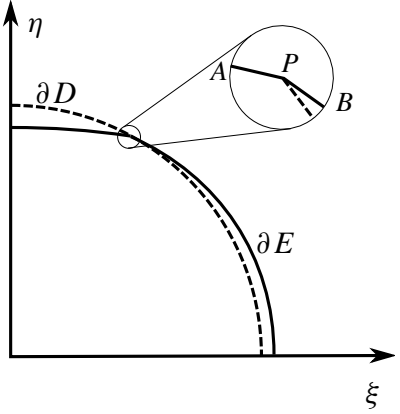

(b)

(c)

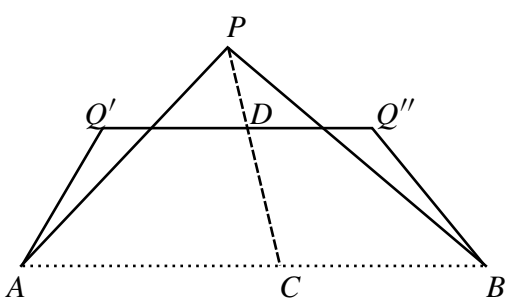

Fig. 5. (a) and (b) correspond to the two possible cases in which $\partial E$ is non-smooth in $P$; and (c) represents a possible way to locally change the shape of $\partial E$, decreasing the isoperimetric deficit without changing the Fraenkel asymmetry index.

The proof can be split into a chain of statements:

(i) $\Omega^{a} \in \mathfrak{S}$;

(ii) $\Omega_{0}^{s}=\Omega^{a}$;

(iii) $\Omega_{0}=\Omega_{0}^{s}$;

(iv) $\Omega=\Omega_{0}$.

These are deduced from well known results, therefore we just sketch their proofs.

Proof of (i). According to Step 3, either $\Omega^{a} \in \mathfrak{S}$ or $\Delta P\left(\Omega^{a}\right)<\Delta P(\Omega)$, but the second case contradicts the hypothesis.

Proof of (ii). Taking into account the proof of Step 2, either $\Omega^{a}=\Omega_{0}^{s}$ or $\Delta P\left(\Omega^{a}\right)<$ $\triangle P\left(\Omega_{0}^{s}\right)$. The second case yields a contradiction.

Proof of (iii). We know that $\Omega_{0}^{s} \in \mathfrak{S}$, hence there exists $0<\bar{\vartheta}<\pi / 4$ such that $\Omega_{0}^{s}=H_{\bar{\vartheta}}$. Two different cases may now occur:

(A) $\bar{\vartheta} \leq \arctan (\pi / 4)$;

(B) $\bar{\vartheta}>\arctan (\pi / 4)$.

In case (A), with the notation of Step $1, \Omega_{0} \cap \Sigma_{\text {out }}=\emptyset$. Arguing as in the proof of Lemma 5.2, taking advantage of (5.4), we show that

$$
\overline{\Omega_{0}}=\{(\xi, \eta): \eta \in[-\bar{d}, \bar{d}], \xi \in[l(\eta), r(\eta)]\},
$$


where $l(\eta)$ and $r(\eta)$, in accordance with Remark 5.2 are locally Lipschitz continuous functions in $(-\bar{d}, \bar{d})$. This is enough to argue as in [28. Section 3.8, proof of (v), p. 117], and deduce that either $\Omega_{0}$ is a translation along the $\xi$-axis of $\Omega_{0}^{s}$, or $\Delta P\left(\Omega_{0}^{s}\right)<\Delta P\left(\Omega_{0}\right)$. But the latter yields a contradiction.

In case (B),

$$
\overline{\Omega_{0} \cap \Sigma_{\text {in }}}=\{(\xi, \eta): \eta \in[-\bar{d}, \bar{d}], \xi \in[l(\eta), r(\eta)]\},
$$

where $l(\eta)$ and $r(\eta)$, in accordance with Remark 5.2, are locally Lipschitz continuous functions in $(-\bar{d}, \bar{d})$. Moreover

$$
\overline{\Omega_{0} \cap D}=\{(\xi, \eta): \xi \in[-1,1], \eta \in[t(\xi), b(\xi)]\},
$$

where $t(\xi)$ and $b(\xi)$, by Remark 5.2, are locally Lipschitz continuous functions in $(-1,1)$. We observe that $\Omega_{0}^{s}$ is the union of two sets: $\overline{\Omega_{0} \cap \Sigma_{\text {in }}}$ Steiner symmetrized with respect to the $\eta$-axis and $\overline{\Omega_{0} \cap D}$ Steiner symmetrized with respect to the $\xi$-axis. Again, arguing as in [28, p. 117], since $\Delta P\left(\Omega_{0}^{s}\right)=\Delta P\left(\Omega_{0}\right)$ we obtain $\Omega_{0}^{s}=\Omega_{0}$.

Proof of (iv). The result easily follows from the characterization of the equality cases in (3.1) and (3.2) stated in Lemma 3.2.

Acknowledgments. This work was financially supported by the MIUR through the PRIN 2006 "Equazioni alle derivate parziali e disuguaglianze funzionali: aspetti quantitativi, proprietà geometriche e qualitative, applicazioni" and by the GNAMPA INDAM.

\section{References}

[1] Alvino, A., Lions, P.-L., Trombetti, G.: On optimization problems with prescribed rearrangements. Nonlinear Anal. 13, 185-220 (1989) Zbl 0678.49003 MR 0979040

[2] Bandle, C.: Isoperimetric Inequalities and Applications. Monogr. Stud. Math. 7, Pitman (Adv. Publ. Program), Boston, MA, and London (1980) Zbl 0436.35063 MR 0572958

[3] Blaschke, W.: Kreis und Kugel. Veit, Leipzig, 1916. JFM 46.1109.01

[4] Bonnesen, T.: Über eine Verschärfung der isoperimetrischen Ungleichheit des Kreises in der Ebene und auf der Kugeloberfläche nebst einer Anwendung auf eine Minkowskische Ungleichheit für konvexe Körper. Math. Ann. 84, 216-227 (1921) JFM 48.0591.03 MR 1512031

[5] Bonnesen, T.: Über das isoperimetrische Defizit ebener Figuren. Math. Ann. 91, 252-268 (1924) JFM 50.0487.03 MR 1512192

[6] Bonnesen, T.: Les problèmes des isopérimètres et des isépiphanes. Collection de monographies sur la théorie des fonctions, Gauthier-Villars, Paris (1929) JFM 55.0431.08

[7] Brothers, J. E., Ziemer, W. P.: Minimal rearrangements of Sobolev functions. J. Reine Angew. Math. 384, 153-179 (1988) Zbl 0633.46030 MR 0929981

[8] Burago, Yu. D., Zalgaller, V. A.: Geometric Inequalities. Grundlehren Math. Wiss. 285, Springer Series in Soviet Math., Springer, Berlin (1988) Zbl 0633.53002 MR 0936419

[9] Chavel, I.: Isoperimetric Inequalities. Differential Geometric and Analytic Perspectives. Cambridge Tracts in Math. 145, Cambridge Univ. Press, Cambridge (2001) Zbl 0988.51019 MR 1849187 
[10] De Giorgi, E.: Sulla proprietà isoperimetrica dell'ipersfera, nella classe degli insiemi aventi frontiera orientata di misura finita. Atti Accad. Naz. Lincei Mem. Cl. Sci. Fis. Mat. Nat. Sez. I (8) 5, 33-44 (1958) Zbl 0116.07901 MR 0098331

[11] Esposito, L., Fusco, N., Trombetti, C.: A quantitative version of the isoperimetric inequality: the anisotropic case. Ann. Scuola Norm. Sup. Pisa Cl. Sci. (5) 4, 619-651 (2005) Zbl 1170.52300 MR 2207737

[12] Figalli, A., Maggi, F., Pratelli, A.: A mass transportation approach to quantitative isoperimetric inequalities. Invent. Math. 182, 167-211 (2010) Zbl pre05782776

[13] Fuglede, B.: Stability in the isoperimetric problem for convex or nearly spherical domains in $\mathbb{R}^{n}$. Trans. Amer. Math. Soc. 314, 619-638 (1989) Zbl 0679.52007 MR 0942426

[14] Fusco, N.: The classical isoperimetric theorem. Rend. Accad. Sci. Fis. Mat. Napoli (4) 71, 63-107 (2004) Zbl 1096.49024 MR 2147710

[15] Fusco, N., Maggi, F., Pratelli, A.: The sharp quantitative isoperimetric inequality. Ann. of Math. (2) 168, 941-980 (2008) Zbl 1187.52009 MR 2456887

[16] Hall, R. R.: A quantitative isoperimetric inequality in $n$-dimensional space. J. Reine Angew. Math. 428, 161-176 (1992) Zbl 0746.52012 MR 1166511

[17] Hall, R. R., Hayman, W. K.: A problem in the theory of subordination. J. Anal. Math. 60, 99-111 (1993) MR 1253231

[18] Hardy, G. H., Littlewood, J. E., Pólya, G.: Inequalities. Reprint of the 1952 edition, Cambridge Math. Library, Cambridge Univ. Press, Cambridge (1988) Zbl 0634.26008 MR 0944909

[19] Hurwitz, A.: Sur le problème des isopérimètres. C. R. Acad. Sci. Paris 132, 401-403 (1901) JFM 32.0386.01

[20] Kawohl, B.: Rearrangements and Convexity of Level Sets in PDE. Lecture Notes in Math. 1150, Springer, Berlin (1985) Zbl 0593.35002 MR 0810619

[21] Kawohl, B.: On the simple shape of stable equilibria. In: Symposia Mathematica, Vol. XXX (Cortona, 1988), Academic Press, London, 73-87 (1989) Zbl 0703.34048 MR 1062606

[22] Lebesgue, H.: Leçons sur les séries trigonométriques professées au Collège de France. Gauthier-Villars, Paris (1906) JFM 37.0281.01 MR 0389527

[23] Osserman, R.: The isoperimetric inequality. Bull. Amer. Math. Soc. 84, 1182-1238 (1978) Zbl 0411.52006 MR 0500557

[24] Osserman, R.: Bonnesen-style isoperimetric inequalities. Amer. Math. Monthly. 86, 1-29 (1979) Zbl 0404.52012 MR 0519520

[25] Stredulinsky, E., P. Ziemer, W.: Area minimizing sets subject to a volume constraint in a convex set. J. Geom. Anal. 7, 653-677 (1997) Zbl 0940.49025 MR 1669207

[26] Talenti, G.: Elliptic equations and rearrangements. Ann. Scuola Norm. Sup. Pisa Cl. Sci. (4) 3, 697-718 (1976) Zbl 0341.35031 MR 0601601

[27] Talenti, G.: Linear elliptic p.d.e.'s: level sets, rearrangements and a priori estimates of solutions. Boll. Un. Mat. Ital. B (6) 4, 917-949 (1985) Zbl 0602.35025 MR 0831299

[28] Talenti, G.: The standard isoperimetric theorem. In: Handbook of Convex Geometry, Vol. A, B, North-Holland, Amsterdam, 73-123 (1993) Zbl 0799.51015 MR 1242977

[29] Toranzos, F. A.: Radial functions of convex and star-shaped bodies. Amer. Math. Monthly 74, 278-280 (1967) Zbl 0145.42802 MR 0208469 